\title{
Scratch no ensino superior: motivação
}

\author{
Rafael Salazar, Valguima Odakura, Carla Barvinski \\ ${ }^{1}$ Faculdade de Ciências Exatas e Tecnologia (FACET) \\ Universidade Federal da Grande Dourados (UFGD) \\ Dourados - MS - Brasil \\ \{salazarafa, valguima.odakura, carlabarvinski\}egmail.com
}

\begin{abstract}
Whereas the initial disciplines of Algorithms and Programming in Higher Education in Computer are the most difficult for students, this article investigates the visual language Scratch as a motivator. An experiment with students was conducted, in which they used the Scratch and were asked about their use. As a result we point out Scratch as a possible motivator.
\end{abstract}

Resumo. Considerando que as disciplinas iniciais de Algoritmos e Programação no Ensino Superior em Computação estão entre as que os alunos mais apresentam dificuldades, investiga-se neste artigo a linguagem visual Scratch como elemento motivador. Foi realizado um experimento com alunos, em que os mesmos utilizaram o Scratch e foram indagados sobre sua utilização. Como resultado apontamos o Scratch como possivel elemento motivador.

\section{Introdução}

O Scratch é uma linguagem de programação visual, desenvolvida pelo grupo Lifelong Kindergarten no Media Lab do MIT para auxiliar na aprendizagem de programação. O Scratch foi lançado publicamente em 2007 [Resnick et al. 2009], [Maloney et al. 2010]. O objetivo de sua criação era o desenvolvimento de uma abordagem de programação que permitisse a qualquer pessoa programar. Dentro desse conceito, o Scratch contém facilidades que permitem a qualquer pessoa sem conhecimentos prévios ou restrição de idade, criar histórias interativas, jogos, animações, simulações, podendo compartilhá-las com sua comunidade, pois o site do Scratch possibilita que os seus usuários compartilhem seus projetos, participando de uma comunidade online de programação.

Considerando as possibilidades do Scratch para o desenvolvimento do racíocinio lógico, bem como o ensino e aprendizagem de programação e as dificuldades dos acadêmicos nas disciplinas iniciais de Algoritmos e Programação em cursos superiores da área de Computação, é razoável explorar o uso do Scratch no ensino de programação para graduação. Neste sentido, [Aureliano and Tedesco 2012], realizaram uma pesquisa com alunos do ensino superior, em disciplinas introdutórias de programação com a intenção de saber se "ensinar programação utilizando o ambiente de programação visual Scratch possibilita um melhor desempenho para os alunos iniciantes em programação". Os resultados de sua pesquisa não apontam para uma melhoria significativa, considerando como desempenho as notas obtidas pelos alunos. Porém, os autores relatam que perceberam melhoria na motivação e participação dos alunos, sendo estes aspectos propostos para avaliação futura. 
Dentro deste contexto, neste artigo objetiva-se investigar a percepção dos alunos do ensino superior ao utilizar a linguagem visual Scratch, considerando o elemento motivação na aprendizagem de Algoritmos e Programação.

Este artigo está organizado como se segue: Na Seção 2 são apresentados os trabalhos relacionados ao uso educacional do Scratch no país. Na Seção 3 é delineado o experimento e são apresentados os resultados. Na Seção 4 os resultados obtidos são discutidos. Por fim, na Seção 5, as considerações finais são apresentadas.

\section{Trabalhos Relacionados}

O Scratch tem sido utilizado com sucesso para fins educacionais no mundo. Para dimensionar sua utilização no Brasil foi realizada uma busca por artigos sobre Scratch na Revista Brasileira de Informática na Educação (RBIE), no Simpósio Brasileiro de Informática na Educação (SBIE) e no Workshop de Informática na Educação (WIE), sendo a RBIE uma revista e o SBIE e WIE eventos nacionais. Todos os três são apoiados pela Comissão Especial de Informática na Educação (CEIE) da Sociedade Brasileira da Computação (SBC).

Tendo como chave de busca a palavra "Scratch", a busca retornou 12 artigos publicados entre 2008 e 2014 nos eventos e revista citados. A Figura 1 ilustra a quantidade de artigos por ano de publicação, sendo possível notar o aumento das publicações sobre o assunto nos últimos anos.

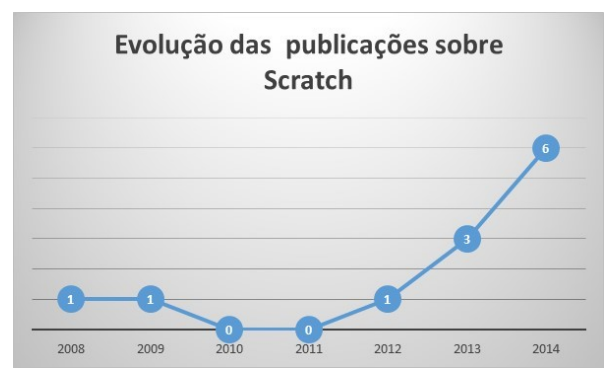

Figure 1. Cenário nacional - quantidade de artigos publicados sobre Scratch por ano.

Na Figura 2 é possível ver a distribuição dos artigos por veículo de publicação, ou seja, RBIE, SBIE e WIE. Percebe-se que o WIE foi o fórum com maior número de artigos sobre o tema, com 8 artigos. Como o WIE é o evento sobre informática na escola e o Scratch foi idealizado para ensinar programação para crianças e adolescentes, é natural que este fórum receba mais publicações sobre o tema. Os números expressam o esforço dos professores em incorporar recursos tecnólogicos que fortaleçam e-ou melhorem processos de aprendizagem.

Ao analisar os 12 artigos, 2 foram eliminados por não tratarem do uso de Scratch e um dos artigos foi publicado 2 vezes, uma no WIE e outra na RBIE. Deste modo, restaram 9 artigos, os quais descrevemos a seguir.

Wangenheim, Nunes e Santos (2014) [von Wangenheim et al. 2014] relatam um estudo de caso sobre o uso do Scratch no Ensino Fundamental em turma de alunos de 6 a 7 anos. Após treinamento de 36 horas com os alunos para o uso do Scratch, foram conduzidas atividades para desenvolver o pensamento computacional, a colaboração mútua 


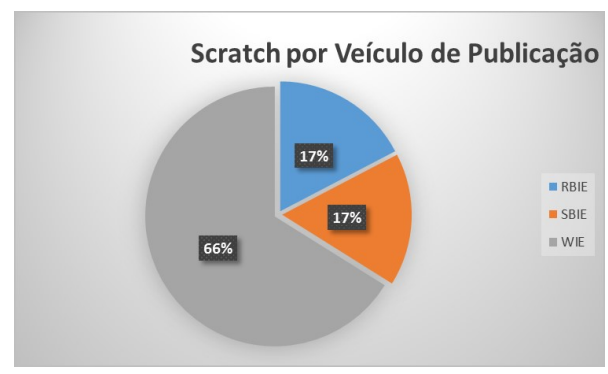

Figure 2. Quantidade de artigos sobre Scratch publicados por veículo (RBIE, SBIE e WIE).

e práticas de programação. Durante o desenvolvimento das atividades os alunos espontaneamente mostravam aos colegas e orientadores seus projetos no Scratch. Ao término do estudo de caso, os alunos externaram interesse em continuar programando com Scratch e vários deles demonstraram tristeza no último encontro do projeto.

Scaico et al (2013) [Scaico et al. 2013] descrevem experiência de uso do Scratch para o ensino de programação no Ensino Médio, com alunos de 15 a 19 anos. Após um treinamento de 20 horas, os alunos participaram de uma olímpiada de programação com Scratch, que avaliou e identificou suas habilidades. A maioria dos alunos, 90\%, conseguiu concluir satisfatoriamente as atividades propostas. Ao final, $80 \%$ dos alunos demonstraram estar motivados a continuar aprendendo a programar após conhecer o Scratch. Um questionário avaliativo apontou que os participantes assimilaram conceitos de programação em relação as estruturas de controle mais comuns em linguagens de programação.

Mota et al (2014) [Mota et al. 2014] apresentam uma proposta de utilização do Scratch para desenvolvimento de raciocínio lógico no Ensino Médio, nas disciplinas de Matemática e Física para resolução de problemas. A proposta contempla um treinamento inicial para os alunos e a aplicação de um questionário no final do projeto para avaliar a utilidade do Scratch para resolução de problemas.

Araújo et al (2013) [de Araújo et al. 2013] abordam a aplicação da taxonomia de Bloom no ensino de programação com Scratch para alunos do Ensino Médio. O treinamento no Scratch durou 16 horas e foi estruturado seguindo a taxonomia de Bloom, que forneceu uma estrutura pedagógica ao curso, em níveis hierárquicos, levando em consideração as categorias de domínio cognitivo: conhecimento, compreensão, aplicação, análise, síntese e avaliação. Ao final do projeto, foi aplicado um questionário em que se pôde observar que as maiores dificuldades dos alunos foram: abstração do problema, planejamento do projeto, entendimento passo a passo do código, raciocínio lógico e começar o projeto. Concluiu-se que o ensino da linguagem Scratch norteada pela taxonomia de Bloom no ensino de programação para alunos no Ensino Médio foi bem sucedida.

Gomes et al (2014) [Gomes et al. 2014] utilizaram o Scratch com alunas do Ensino Médio com a intenção de incentivar meninas para a área da Computação. O projeto trabalhou com 3 meninas que receberam treinamento de 10 horas e depois trabalharam com programação nas aulas de Química, Física e Matemática, desenvolvendo animações. Ao final dos projetos as alunas relataram que utilizar o Scratch é algo possível e consideraram a ferramenta fácil, legal, divertida e instigante. 
Arantes, Amiel e Fedel (2014) [Arantes et al. 2014] relataram um projeto com jovens de 16 a 18 anos, em que o Scratch foi apresentado com o objetivo de desenvolver a autonomia tecnológica entre os jovens. Após treinamento de 12 horas, os participantes conseguiram desenvolver satisfatoriamente o jogo proposto. Ao final do projeto, todos os alunos disseram que gostaram do contato com a programação e a maioria demonstrou interesse em participar de outras oficinas.

Silva, Moraes e Batista (2014) [Silva et al. 2014] relatam as percepções de alunos e professores no uso de Scratch para a criação de objetos de aprendizagem sobre saneamento básico. O projeto foi desenvolvido em uma turma de quinta série do Ensino Fundamental. Ao término do projeto, os autores concluíram que o uso do Scratch contribuiu para a ampliação do conhecimento dos alunos sobre saneamento básico.

Silva, Araújo e Aranha (2014) [da Silva et al. 2014] realizaram oficinas de 4h de Scratch para professores do Ensino Médio e Fundamental. Os autores relataram que as oficinas foram bem aceitas pelos professores e que as atividades aplicadas auxiliaram no aprendizado de fundamentos da computação. Além disso, apontaram que o Scratch pode fortalecer os vínculos de interação entre professores e alunos de forma lúdica e interativa, reforçando a troca de conhecimentos decorrentes dessas interações.

França e Amaral (2013) [de França and do Amaral 2013] descrevem a aplicação de uma oficina de 3 horas de Scratch para alunos de 13 a 14 anos. Durante a oficina os alunos puderam explorar vários comandos e conceitos computacionais. Através dos projetos desenvolvidos, eles puderam demonstrar competência em conceitos básicos de programação.

O conjunto de artigos encontrado sobre Scratch forma um panorama dos trabalhos realizados pela comunidade de Informática na Educação no país. Todos os artigos utilizam o Scratch no Ensino Fundamental e Médio e relatam bons resultados, tanto no que tange o aprendizado de programação como na motivação e interesse dos alunos. Observase também, que o treinamento inicial dos alunos com Scratch variou entre 3 e 36 horas e que as aplicações foram tanto em disciplinas curriculares da área de exatas, como Física, Química e Matemática, como em assuntos de interesse geral, como saneamento básico.

\section{Experimento}

Nesse artigo, busca-se responder se a utilização do Scratch no ensino superior pode auxiliar na aprendizagem de Algoritmos e Programação. Para responder essa pergunta, ao invés de experimentarmos o uso de Scratch em disciplinas iniciais de Algoritmos e Programação, este foi apresentado para alunos que já cursaram tais disciplinas. No experimento, os alunos conheceram a ferramenta e depois avaliaram se gostariam ou não de a ter utilizado nas disciplinas de Algoritmos e Programação e se esta utilização impactaria na sua motivação.

O experimento conduzido foi delineado da seguinte forma: a) um questionário inicial para verificar as impressões dos alunos sobre as disciplinas iniciais de Algoritmos e Programação que eles já cursaram. b) a apresentação e utilização do Scratch. c) um questionário final para identificar a percepção deles quanto a motivação na utilização do Scratch.

Após a aplicação do questionário inicial, os alunos participaram de uma aula de 50 
minutos em que o Scratch foi apresentado e foram ilustrados seus conceitos e ferramentas. Após essa aula, os alunos utilizaram 50 minutos para resolver problemas usando o Scratch com o auxílio do professor.

No experimento participaram 22 alunos do curso de Bacharelado em Sistemas de Informação, os quais já cursaram as disciplinas iniciais de Algoritmos e Programação. Destes, $91 \%$ (20) são do sexo masculino e 9\% (2) são do sexo feminino, sendo esta proporção uma realidade nos cursos de graduação em Computação. A faixa etária desses alunos está distribuída da seguinte forma: 32\% (7) tem menos que 20 anos, 36\% (8) estão entre 21 e 24 anos e $32 \%$ (7) tem entre 25 e 30 anos. Como o curso de Sistemas de Informação é em créditos, estes alunos estão em períodos acadêmicos diferentes, porém todos já cursaram pelo menos uma vez, as disciplinas em questão.

\subsection{Questionário inicial}

O questionário inicial teve como objetivo geral obter informações sobre a experiência dos alunos com as disciplinas de Algoritmos e de Programação, sua aprovação nessas disciplinas, o grau de importância que os alunos atribuem as mesmas e a motivação para cursá-las.

Questionados sobre a importância das disciplinas para sua formação acadêmica, conforme ilustra a Figura 3, a maioria, 91\% (20), afirmou que tais disciplinas são fundamentais, enquanto $4.5 \%$ (1) acreditam que as disciplinas são relevantes e $4.5 \%$ (1) consideram que as disciplinas são desnecessárias. A análise dos dados apresentados indica o nível de maturidade dos acadêmicos ao considerar as referidas disciplinas como fundamentais para sua formação.

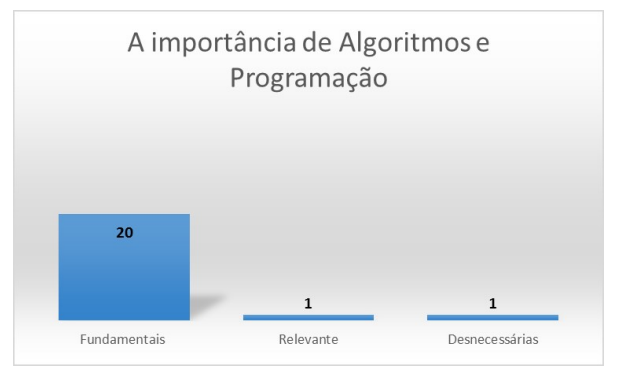

Figure 3. A visão dos alunos sobre a importância das disciplinas de Algoritmos e Programação em sua formação acadêmica.

Indagados sobre sua motivação ao cursarem as disciplinas, 63.6\% (14) estavam muito motivados, $31.8 \%$ (7) estavam motivados e $4.5 \%$ (1) estavam desmotivados, conforme ilustra Figura 4. Desta forma, nota-se que a maioria dos alunos 95\% (21) estavam motivados para cursar as disciplinas.

Ainda, os alunos foram convidados a expressar sua motivação para aprender Algoritmos e Programação. A questão foi aberta e as respostas obtidas foram classificadas em 2 categorias: prática didática-metodológica do professor e os recursos. $\mathrm{O}$ aspecto considerado pelos alunos como sendo o mais relevante para sua motivação nas disciplinas foi a didática e a metodologia do professor. De acordo com os alunos, um professor com uma boa didática, empenhado em ensinar, que apresente exemplos bem estruturados e claros, que utilize exercícios desafiadores e dinâmicos afeta sua motivação para o estudo. 
CBIE-LACLO 2015

Anais do XXVI Simpósio Brasileiro de Informática na Educação (SBIE 2015)

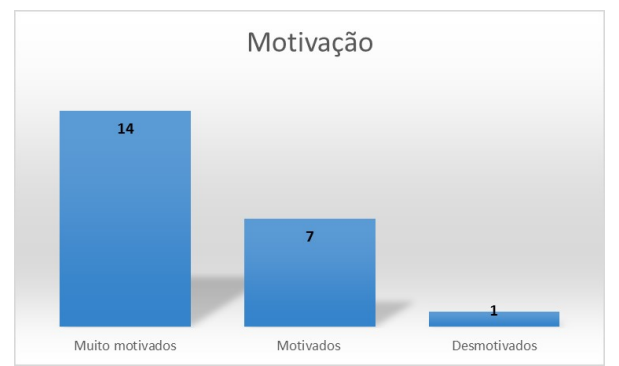

Figure 4. O grau de motivação dos alunos ao cursar as disciplinas de Algoritmos e Programação.

Com relação ao item recurso, os alunos elencaram a necessidade de mais aulas práticas em laboratório. A Figura 5 resume os aspectos que impactam na motivação. A categoria Outros inclui respostas como "nenhum fator" e a contextualização das disciplinas na vida acadêmica e profissional.

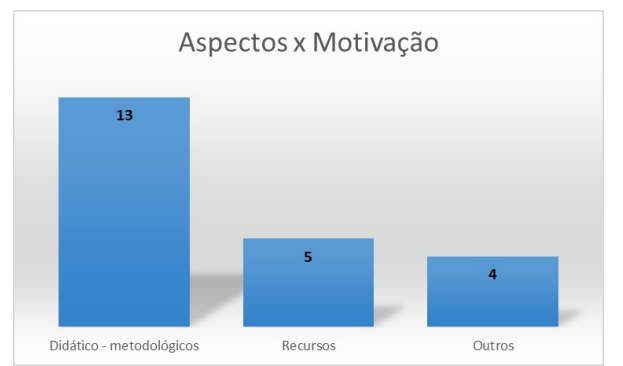

Figure 5. Aspectos que impactam na motivação dos alunos.

Perguntados se gostam de programar, $45.5 \%$ (10) responderam que gostam muito, $50 \%$ (11) mais ou menos e $4.5 \%$ (1) de jeito nenhum, conforme ilustra Figura 6. Verificase que há alunos totalmente sem afinidade com o curso, considerando a importância da programação em um curso de Computação. Por outro lado, o fato de 50\% apreciar mais ou menos a programação, requer adoção de práticas que os estimulem a gostar de programação.

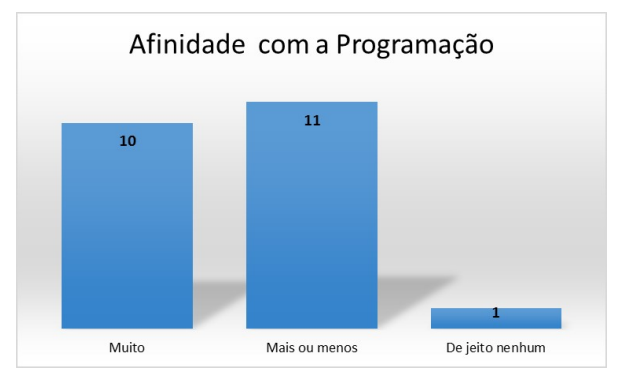

Figure 6. Afinidade com programação.

A aprovação na disciplina de Algoritmos teve a seguinte distribuição: 63.7\% (14) passaram na primeira vez que cursaram a disciplina, $18.2 \%$ (4) passaram na segunda tentativa e $18.2 \%$ (4) ainda estão tentando ser aprovados. O aproveitamento na disciplina de Algoritmos pode ser visto na Figura 7. 
CBIE-LACLO 2015

Anais do XXVI Simpósio Brasileiro de Informática na Educação (SBIE 2015)

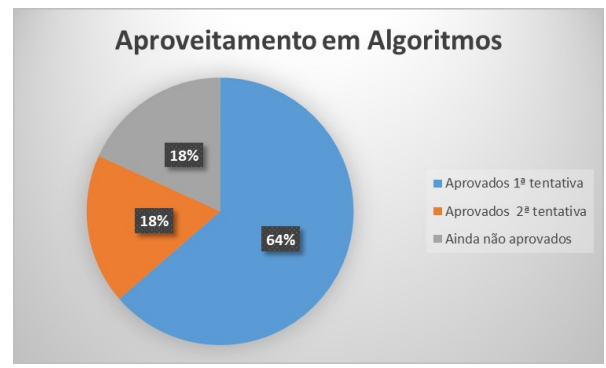

Figure 7. Aproveitamento na disciplina de Algoritmos.

Dos alunos aprovados na primeira tentativa em Algoritmos, apenas 29\% (4) relataram que passaram com muito esforço e $71 \%$ (10) passaram com facilidade.

A aprovação na disciplina de Programação teve a seguinte distribuição: $68.2 \%$ (15) passaram na primeira vez que cursaram a disciplina, $13.6 \%$ (3) passaram na segunda tentativa e $18.2 \%$ (4) ainda estão tentando ser aprovados. O aproveitamento na disciplina de Programação pode ser visto na Figura 8.

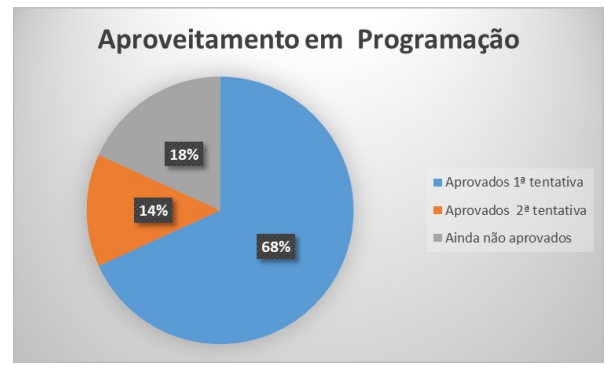

Figure 8. Aproveitamento na disciplina de Programação.

Dos alunos aprovados na primeira tentativa em Programação, apenas 27\% (4) relataram que passaram com muito esforço e $73 \%$ (11) disseram que passaram com facilidade.

Indagados sobre os obstáculos no aprendizado das disciplinas de Algoritmos e Programação os alunos responderam: $30 \%$ (8) que o conteúdo era abstrato demais, $22 \%$ (7) que não entendia a sintaxe, $26 \%$ (6) que os exemplos eram poucos e $22 \%$ (7) que não entendia a lógica de programação. Os obstáculos no aprendizado podem ser visualizados na Figura 9.

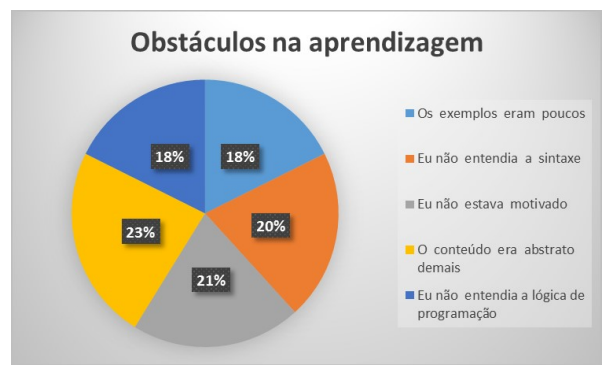

Figure 9. Obstáculos na aprendizagem. 
CBIE-LACLO 2015

Anais do XXVI Simpósio Brasileiro de Informática na Educação (SBIE 2015)

\subsection{Questionário final}

Após a utilização do Scratch os alunos responderam um questionário final aplicado com o objetivo de conhecer a percepção dos mesmos em relação ao aprendizado de Algoritmos e Programação auxiliado pelo Scratch. Dos 22 alunos participantes do primeiro questionário, apenas 21 responderam o segundo questionário.

A primeira pergunta foi se os alunos já conheciam o Scratch antes, 90.5\% (19) disseram que o desconheciam, enquanto 9.5\% (2) disseram já ter usado o Scratch anteriormente.

Sobre a utilidade do Scratch para o aprendizado nas disciplinas de Algoritmos e Programação, 71\% (15) relataram que a ferramenta seria muito útil, 10\% (2) consideraram a ferramenta útil, enquanto $19 \%$ (4) acharam a ferramenta pouco útil. A percepção dos alunos sobre a utilização do Scratch está resumida na Figura 10.

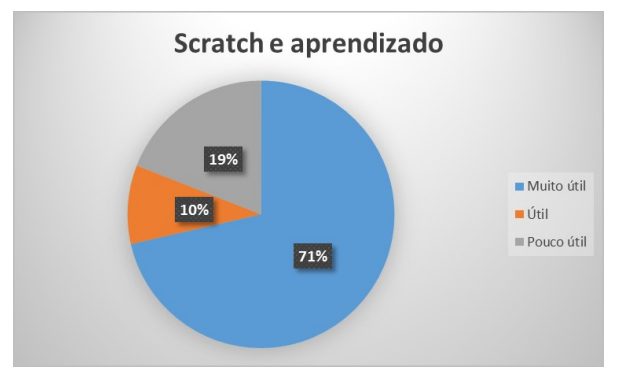

Figure 10. Visão dos alunos sobre a utilidade do Scratch para a aprendizagem em Algoritmos e Programação.

Os alunos que gostariam de ter aprendido Algoritmos e Programação com Scratch representam $38.1 \%$ (8), enquanto $61.9 \%$ (13) mostraram-se indiferentes quanto à sua utilização no aprendizado.

Ainda, foi solicitado aos alunos que comparassem suas aulas práticas de laboratório de programação com a experiência que tiveram com o Scratch: 72\% (18) consideram que o Scratch facilitaria entender os conceitos de algoritmos antes de iniciar programação com uma linguagem específica, 24\% (6) consideram que o Scratch é mais empolgante, mas insuficiente para aprendizado de programação e 4\% (1) consideram que o Scratch poderia substituir a sintaxe das linguagens de programação vistas no curso. Na Figura 11 é possível visualizar as informações relativas a experiência dos alunos com o Scratch.

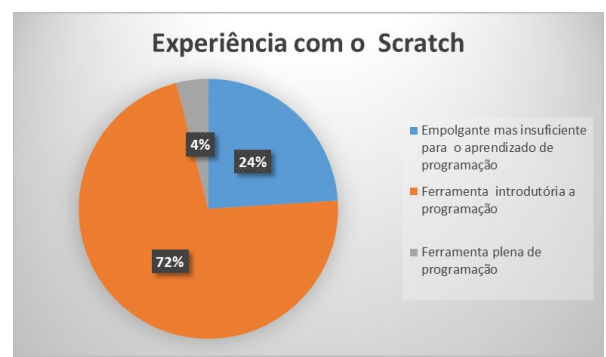

Figure 11. Visão dos alunos sobre o Scratch como linguagem de Programação. 


\section{Discussão}

Ao confrontarmos os dados da motivação ilustrados na Figura 4, com os dados da aprovação apresentados na Figura 7, é possível visualizar a relação entre a motivação do aluno e sua aprovação na disciplina de Algoritmos. O resultado principal é que a motivação é fator importante na aprovação. Os dados demonstram que os alunos "muito motivados" tem maior aprovação na disciplina quando comparados com os alunos "motivados" e "pouco motivados", conforme ilustram as Figuras 12(a) e 12(b). Ressalta-se que nas Figuras 12(a) e 12(b) não são apresentados dados para a categoria "pouco motivado", uma vez que essa categoria tem apenas um aluno aprovado na segunda tentativa. Nota-se também que os resultados de aprovação e motivação são semelhantes para a disciplina de Programação e por essa razão foram aqui omitidos.

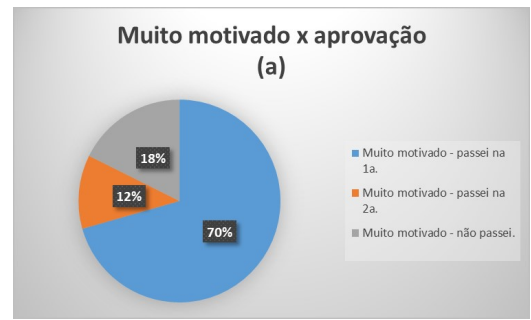

(a) Muito motivados

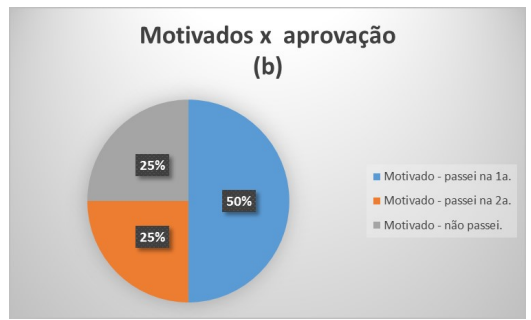

(b) Motivados

Figure 12. Relação entre alunos: (a) muito motivados e aprovação e (b) motivados e aprovação.

Todavia, observou-se que a motivação não é fator determinante para aprovação, sendo necessário aprofundar investigações sobre outros aspectos apontados como obstáculos na aprendizagem conforme a Figura 9. É necessário detectar que elementos estão impedindo a aprovação de alunos que apesar de "muito motivados" ou "motivados" terminam por reprovar. Um ponto de partida pode ser o cruzamento dos obstáculos para aprendizagem ilustrados na Figura 9, com os dados relacionados com a motivação e a aprovação, mostrados na Figura 12, pois essas informações podem auxiliar na percepção de outras dimensões que afetam o aprendizado e por sua vez o aproveitamento dos alunos nas disciplinas em foco.

Os aspectos que impactam a motivação e os obstáculos para a aprendizagem, respectivamente nas Figuras 5 e 9, explicitam em maiores detalhes as necessidades de constante revisão e aperfeiçoamento dos aspectos didático-metodológicos utilizados pelo professor, à medida em que aponta os aspectos que prejudicaram um melhor aprendizado por parte dos alunos.

Ao introduzir comparações entre os obstáculos para a aprendizagem de programação e a utilidade do Scratch para tal aprendizado, ilustrados respectivamente pelas Figuras 9 e 10, pode-se considerar que o Scratch poderia ser instrumento, ainda que parcial, para solução da problemática didático-metodológica apontada anteriormente. Todavia, analisando a visão dos alunos sobre o uso do Scratch, na Figura 11, verifica-se que $96 \%$ deles destacam a insuficiência do Scratch como ferramenta de aprendizado para as disciplinas de Algoritmos e Programação em séries iniciais de um curso superior de Computação. 


\section{Considerações Finais}

Neste artigo são apresentados resultados sobre o uso de Scratch para ensino e aprendizado de Algoritmos e Programação num curso de graduação em Computação. Os resultados apontam que o Scratch não é suficiente para aprendizagem de programação em disciplinas de graduação, mas que sua utilização na introdução dos conceitos, em conjunto com linguagens de programação tradicionais, pode aumentar a motivação do aluno e consequentemente favorecer seu aprendizado.

Como trabalhos futuros, faz-se necessário pesquisas aprofundadas sobre as relações entre a atuação didático-metodológica do professor, a motivação do aluno e as ferramentas que apoiam o processo de ensino e aprendizagem.

\section{References}

Arantes, F. L., Amiel, T., and Fedel, G. (2014). Nos rumos da autonomia tecnológica-desafios e lições aprendidas para a formação de jovens. In Anais do Workshop de Informática na Escola, volume 20.

Aureliano, V. C. O. and Tedesco, P. d. A. (2012). Avaliando o uso do scratch como abordagem alternativa para o processo de ensino-aprendizagem de programação. In Congresso da Sociedade Brasileira da Computação, volume 32, pages 1-10.

da Silva, T. R., de Araujo, G. G., and da Silva Aranha, E. H. (2014). Oficinas itinerantes de scratch e computação desplugada para professores como apoio ao ensino de computação-um relato de experiência. In Anais do Workshop de Informática na Escola, volume 20.

de Araújo, A. L. S. O., Scaico, P. D., de Paiva, L. F., de Morais Rabêlo, H., de Luna Santos, L., Pessoa, F. I. R., Targino, J. M., and dos Santos Costa, L. (2013). Aplicação da taxonomia de bloom no ensino de programação com scratch. In Anais do Workshop de Informática na Escola, volume 1.

de França, R. S. and do Amaral, H. J. C. (2013). Proposta metodológica de ensino e avaliação para o desenvolvimento do pensamento computacional com o uso do scratch. In Anais do Workshop de Informática na Escola, volume 1.

Gomes, W. F., Louzada, C. S., Nunes, M. A. S. N., Salgueiro, E. M., and Andrade, B. T. (2014). Incentivando meninas do ensino médio à área de ciência da computação usando o scratch como ferramenta. In Anais do Workshop de Informática na Escola, volume 20.

Maloney, J., Resnick, M., Rusk, N., Silverman, B., and Eastmond, E. (2010). The scratch programming language and environment. ACM Transactions on Computing Education (TOCE), 10(4):16.

Mota, F. P., Ribeiro, N. F., Emmendorfer, L., Butzen, P., Machado, K. S., and Adamatti, D. F. (2014). Desenvolvendo o raciocínio lógico no ensino médio: uma proposta utilizando a ferramenta scratch. In Anais do Simpósio Brasileiro de Informática na Educação, volume 25, pages 377-381.

Resnick, M., Maloney, J., Monroy-Hernández, A., Rusk, N., Eastmond, E., Brennan, K., Millner, A., Rosenbaum, E., Silver, J., Silverman, B., et al. (2009). Scratch: programming for all. Communications of the ACM, 52(11):60-67.

Scaico, P. D., de Lima, A. A., Azevedo, S., da Silva, J. B. B., Raposo, E. H., Alencar, Y., Mendes, J. P., Scaico, A., et al. (2013). Ensino de programação no ensino médio: Uma abordagem orientada ao design com a linguagem scratch. Revista Brasileira de Informática na Educação, 21(02):92.

Silva, A. M. d. S., Moraes, D. A. S. d. S., and Batista, S. C. F. (2014). Objetos de aprendizagem em scratch para estudo de saneamento básico: Percepções de alunos e professores. In Anais do Workshop de Informática na Escola, volume 20.

von Wangenheim, C. G., Nunes, V. R., and dos Santos, G. D. (2014). Ensino de computação com scratch no ensino fundamental-um estudo de caso. Revista Brasileira de Informática na Educação, 22(03):115. 